\title{
On Stability and Passivity of a Class of Hybrid Systems
}

\author{
A. Yu. Pogromsky, M. Jirstrand, and P. Spångéus \\ Department of Electrical Engineering \\ Linköping University, S-581 83 Linköping, Sweden \\ WWW: http://www. control.isy.liu.se \\ Email: \{sasha,matsj, per\}@isy.liu.se
}

March 4, 1998

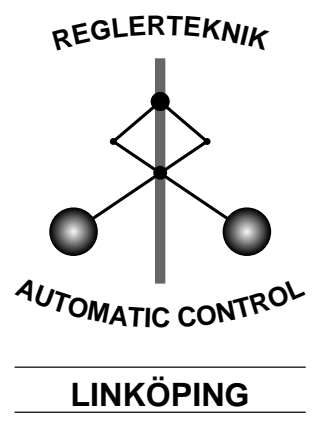

\section{Report no.: LiTH-ISY-R-2017 \\ Submitted to CDC98}

Technical reports from the Automatic Control group in Linköping are available by anonymous ftp at the address ftp.control.isy.liu.se. This report is contained in the compressed postscript file 2017.ps.z. 



\title{
On Stability and Passivity of a Class of Hybrid Systems
}

\author{
A. Yu. Pogromsky*, M. Jirstrand, and P. Spångéus \\ Department of Electrical Engineering \\ Linköping University, 58183 Linköping, Sweden \\ www: www.control.isy.liu.se \\ email: $\{$ sasha,mats j,per\}@isy.liu.se
}

\begin{abstract}
The paper deals with the stabilizability and passifiability properties of a class of hybrid dynamical systems. The systems under consideration are composed of a continuous time invariant plant and discrete event controller. An algebraic criterion for existence of a Lyapunov function for a piecewise linear system is given. Based on these results some passifiability issues are considered.
\end{abstract}

Keywords: Hybrid dynamical systems, controlled switching, passivity

\section{Introduction}

Recently, a remarkable attention has been drawn to the problem of stability/stabilizability of the so-called hybrid dynamical systems (HDS) (see $[1,2,3,4]$ and references therein). The most common view of hybrid systems is to consider them as systems with different combinations of discrete and continuous behavior, or systems that can make discrete jumps between different continuous behaviors. This concept of hybrid systems is very close to the concept of variable structure systems (VSS). In [5, page 39] this concept was described as follows: variable structure systems are systems in which

*On leave from the Institute for Problems of Mechanical Engineering, 61, Bolshoy, VO, St.Petersburg, 199178, Russia 
connections between elements are subject to change depending on the system state. For example, any continuous computer controlled process can be considered both as HDS and VSS. However, nowadays the concept of VSS is mostly associated with systems having sliding modes.

In this paper we consider a class of HDS (or a class of VSS) which can be specified as follows: given a continuous-time plant and a set of different continuous controllers, during the system evolution it can be switched between the controllers according to some switching rules in order to achieve a desired performance. This concept of controlled switching was introduced in $[6,2]$. This class of systems can demonstrate rather interesting phenomena. For example, in [3] it was shown that the trivial solution of HDS can be stabilized by appropriate switchings between the basic controllers even in case when none of the basic controllers can stabilize the system alone.

Now piecewise quadratic Lyapunov functions have been studied by different authors. For example, in [4] it was demonstrated that the search for a piecewise quadratic Lyapunov function for analysis of stability can be stated as a convex optimization problem in terms of linear matrix inequalities which can be effectively solved by numerical methods $[7,8]$. In this paper we will use piecewise quadratic Lyapunov functions in order to give sufficient conditions of stabilizability of systems via controlled switching. Our problem is a problem of design. Hence, the results in [4] cannot be employed in our case. We will present a constructive criterion that allows us to answer both questions: whether the system is stabilizable via controlled switching and how to find an appropriate switching rule which stabilizes the system at the origin.

Of course, the most direct application of Lyapunov functions lies in the field of stability analysis and controller design. However, different notions of the theory of dissipative systems, e.g. storage, energy functions, are very close to Lyapunov functions. With this in mind it is interesting to introduce concepts such as passivity/passifiability into the realm of hybrid systems. This attempt is also made in the present paper. This allows one to employ the well developed passivity based technique (see e.g. [11]) to design switching rules for hybrid dynamical systems. Another important reason to introduce passivity is to tackle the complexity of hybrid systems. In applications many hybrid systems have a hierarchical structure where switching might occur at many levels. Establishing passivity for subsystems, which themselves might be hybrid, would simplify stability/performance analysis of the whole system since we can then utilize the many results on interconnections of passive systems, see e.g. [11].

The paper is organized as follows. First we pose the statement of the problem of stabilizability and passifiability of systems via controlled switch- 
ing. In Section 4 we present sufficient conditions of stabilizability of linear systems via controlled switching with linear basic controllers. In Section 5 we give an algebraic criterion of passifiability of systems via controlled switching using results on existence of Lyapunov functions.

\section{Preliminaries}

In the paper we use the following notations. The Euclidean norm in $\mathbb{R}^{n}$ is denoted as $|\cdot|,|x|^{2}=x^{\top} x$, where ${ }^{\top}$ stands for the transpose operation. A function $V: X \rightarrow \mathbb{R}_{+}$defined on a subset $X$ of $\mathbb{R}^{n}, 0 \in X$ is positive definite if $V(x)>0$ for all $x \in X \backslash\{0\}$ and $V(0)=0$. It is radially unbounded if $V(x) \rightarrow \infty$ as $|x| \rightarrow \infty$.

Consider the following nonlinear time-invariant system

$$
\dot{x}=f(x),
$$

where $x(t) \in \mathbb{R}^{n}$ and $f$ satisfies some assumptions guaranteeing the existence of a unique solution in some reasonable sense at least on a finite time interval. The derivative of the function $V$ with respect to the vector field $f$ calculated at the point $x$ is denoted $L_{f} V(x): L_{f} V(x)=(\partial V(x) / \partial x) f(x)$. In this paper we will also use the same notation for functions $V$ which are not $\mathcal{C}^{1}$-smooth but have derivatives in any direction. In this case we denote

$$
\left.L_{f} V(x)=\lim _{\delta \rightarrow 0_{+}} \delta^{-1}(V(x+\delta f(x))-V(x))\right) .
$$

\section{Problem Statement}

Consider the following system

$$
\left\{\begin{array}{l}
\dot{x}=f(x)+g(x) u+g_{1}(x) v \\
y=h(x)
\end{array}\right.
$$

where $x(t) \in \mathbb{R}^{n}$ is the state and $u(t) \in \mathbb{R}^{m}$ is the control, $v(t) \in \mathbb{D} \subset \mathbb{R}^{l}$ is an additional input, which is assumed to be locally square integrable, the set of admissible additional input functions will be denoted as $\mathcal{V}$ and $y(t) \in \mathbb{R}^{l}$ is the system output. The vector functions $f, g, h$ are assumed to be smooth enough to ensure existence and uniqueness of all solutions of the differential systems considered below in some reasonable sense, e.g. in the sense of Filippov, $f(0)=0, h(0)=0$.

Suppose we have a collection of given feedback controllers

$$
u_{1}=\phi_{1}(x), u_{2}=\phi_{2}(x), \ldots, u_{k}=\phi_{k}(x),
$$


where $\phi_{r}, r \in \mathbb{N}, \mathbb{N}=\{1,2, \ldots, k\}$ are some given vector functions. Following $[2,3]$ the controllers in $(3)$ are called basic controllers. The control law is determined by a switching function $\mathcal{I}: \mathbb{R}^{n} \rightarrow \mathbb{N}$ which defines the index of the basic controller to be applied at the time instant $t$ according to the on-line measurements of the system states:

$$
u(t)=\phi_{i(t)}(x(t)), \quad i(t)=\mathcal{I}(x(t)) .
$$

Consider the following problem: how to find an appropriate switching rule such that the closed loop system is

1) globally asymptotically stable with zero input $v$;

2) passive with respect to input $v$ and output $y$.

One can consider also a dual problem: given a switching function $\mathcal{I}: \mathbb{R}^{n} \rightarrow$ $\mathbb{N}$, the problem is to analyze stability/passivity of the closed loop system. These problems can be formalized by the following definitions.

Definition 1 The system (2) with $v \equiv 0$ is said to be stabilizable via controlled switching with the basic controllers (3) if there exists a switching function $\mathcal{I}: \mathbb{R}^{n} \rightarrow \mathbb{N}$ such that the trivial solution of the closed loop system (2), (3), (4) is globally asymptotically stable.

Since we are looking for the conditions guaranteeing global stability of the trivial solution we will say with a little abuse of terminology that the controller with switchings stabilizes the system when it stabilizes its trivial solution.

Definition 2 The system (2) is said to be passifiable via controlled switching with the basic controllers (3) if for some switching function $\mathcal{I}: \mathbb{R}^{n} \rightarrow \mathbb{N}$ there exists a nonnegative function $V: \mathbb{R}^{n} \rightarrow \mathbb{R}_{+}, V(0)=0$ such that the following dissipation inequality is satisfied along trajectories of the closed loop system (2), (3), (4).

$$
V(x(t))-V(x(0)) \leq \int_{0}^{t} h(x(s))^{\top} v(s) d s
$$

for all $v(t) \in \mathcal{V}, x_{0} \in \mathbb{R}^{n}, 0 \leq t<T_{x_{0}, v, \mathcal{I}}$, where $T_{x_{0}, v, \mathcal{I}}$ is the upper time limit for which the solution of the closed loop system with initial conditions $x(0)=x_{0}$ exists.

If this inequality is modified as follows

$$
V(x(t))-V(x(0)) \leq \int_{0}^{t} h(x(s))^{\top} v(s) d s-\int_{0}^{t} S(x(s)) d s,
$$

where $S$ is a positive definite function then the system (2) is referred to as state strictly passifiable. Finally, if $S=S_{1} \circ h$ and $S_{1}$ is positive definite then the system (2) is called output strictly passifiable. 


\section{On Stabilization via Controlled Switching}

First we give the following definition which is motivated by the one given in $[3]$.

Definition 3 Given a collection $\left\{z_{1}, z_{2}, \ldots, z_{k}\right\}$ of scalar functions $z_{r}: \mathbb{R}^{n} \rightarrow$ $\mathbb{R}$. Let $\Omega_{r}:=\left\{x \in \mathbb{R}^{n}: z_{r}(x) \leq-S(x)\right\}$ where $S: \mathbb{R}^{n} \rightarrow \mathbb{R}$ is some continuous scalar function. The collection $\left\{z_{1}, z_{2}, \ldots, z_{k}\right\}$ is called complete (resp. strictly complete) if $S$ is nonnegative (resp. positive definite) and $\cup_{r} \Omega_{r}=\mathbb{R}^{n}$. If $\cup_{r} \Omega_{r}=\Omega \subset \mathbb{R}^{n}$ the collection is called complete (resp. strictly complete) in $\Omega$.

If the function $S$ in Definition 3 takes the form $S=S_{1} \circ h$ and $S_{1}$ is positive definite then the collection $\left\{z_{1}, z_{2}, \ldots, z_{k}\right\}$ is referred to as strictly complete with respect to the mapping $h$.

Using the direct Lyapunov method it is not difficult to notice that the system (2) with $v \equiv 0$ is stabilizable via controlled switching if there exists a positive definite radially unbounded function $V: \mathbb{R}^{n} \rightarrow \mathbb{R}_{+}$such that the collection

$$
\left\{L_{f+g \phi_{1}} V(x), \ldots, L_{f+g \phi_{k}} V(x)\right\}
$$

is strictly complete.

Now let us establish conditions of stabilizability of the linear system

$$
\dot{x}=A x+B u
$$

via the controlled switching

$$
u(t)=K_{i(t)} x(t), \quad i(t)=\mathcal{I}(x(t)) .
$$

with the basic controllers defined as

$$
u_{1}=K_{1} x, u_{2}=K_{2} x, \ldots, u_{k}=K_{k} x
$$

\subsection{Quadratic Lyapunov Functions}

First we consider the case when the Lyapunov function which proves stability of the closed loop system is a quadratic form.

In [3] it was found that for quadratic stabilizability of linear systems via controlled switching with linear basic controllers it is necessary and sufficient that the corresponding collection of quadratic forms is strictly complete:

Theorem 1 The two following statements are equivalent: 
I. There exists a switching function $\mathcal{I}: \mathbb{R}^{n} \rightarrow \mathbb{N}$ which exponentially stabilizes the system (6), (8), (7) with a quadratic Lyapunov function $V: \mathbb{R}^{n} \rightarrow \mathbb{R}_{+}$.

II. There exist a positive definite matrix $P=P^{\top}>0$ such that the collection of quadratic forms $\left\{L_{A+B K_{1}} V(x), L_{A+B K_{2}} V(x) \ldots, L_{A+B K_{k}} V(x)\right\}$ with $V=x^{\top} P x$ is strictly complete with a quadratic function $S$.

It is worth mentioning that if we are interested in asymptotic stabilizability then this result may be conservative since the requirement of existence of a quadratic Lyapunov function may be too restrictive. Indeed in [4] it was shown that there is a simple 2nd order hybrid system with asymptotically stable zero equilibrium but there is no quadratic form which can be taken as a Lyapunov function for this system. Therefore, at least for the problem of stability analysis it is preferable to enlarge the possible class of Lyapunov functions. In the sequel, we will find necessary and sufficient conditions of stabilizability for a wider class of piecewise quadratic Lyapunov functions.

\subsection{Convex Homogeneous Lyapunov functions}

Now let us formulate stabilizability conditions for a wider class of Lyapunov functions. We will say that a positive definite function $V: \mathbb{R}^{n} \rightarrow \mathbb{R}_{+}$differentiable in all directions belongs to a class $\mathcal{H C}$ if it is convex and homogeneous of degree two. It is clear that any $\mathcal{H C}$-function is radially unbounded. Notice also that the sets of the form $\left\{x \in \mathbb{R}^{n}: V(x) \leq C, C>0\right\}$ of the $\mathcal{H C}$-functions are convex centrally symmetric bodies and therefore they can be approximated with arbitrarily desired accuracy by centrally symmetric convex polytopes. If the number of vertices of the approximating polytope is large enough then we can choose a new $\mathcal{H C}$-function with a level surface that coincides with the boundary of the approximating polytope such that the derivative of the approximated function in the direction defined by some vector field has the same sign as the derivative in the same direction of the approximating function. Therefore in the class of $\mathcal{H C}$-Lyapunov functions it is sufficient to consider only functions with level surfaces which are boundaries of centrally symmetric convex polytopes. The following theorem formalizes this statement.

Theorem 2 The two following statements are equivalent:

I. There exists a switching function $\mathcal{I}: \mathbb{R}^{n} \rightarrow \mathbb{N}$ which exponentially stabilizes the system (6), (8), (7) with a $\mathcal{H C}$-Lyapunov function. 
II. There exist a full rank matrix $\mathcal{L}=\left(l_{1}, l_{2}, \ldots, l_{M}\right)$ with $M \geq n$ and an $\mathcal{H C}$-Lyapunov function in the form

$$
V(x)=\max _{1 \leq j \leq M}\left(l_{j}^{\top} x\right)^{2}
$$

such that the collection

$$
\left\{L_{A+B K_{1}} V(x), L_{A+B K_{2}} V(x) \ldots, L_{A+B K_{k}} V(x)\right\}
$$

is strictly complete with a quadratic function $S$.

Proof: $\quad(\mathrm{I}) \Longrightarrow(\mathrm{II})$. With the help of arguments in [9, Theorem 2], one can approximate the level surfaces of the $\mathcal{H C}$-function $W$ by centrally symmetrical convex polytopes which form the level surfaces of the function (9) (due to the rank condition). Observe that if the approximated and approximating function coincide at a point $x$ they coincide for any point $\alpha x, \alpha \in \mathbb{R}$ since both functions are homogeneous of the same degree. Hence, a centrally symmetric polytope that approximates a level set of the approximated function, defines an approximating function whose level sets approximates all level sets of the approximated function. By choosing $M$ large enough (but finite, see [9]) one can achieve that the time derivative of $V$ is negative and satisfies a quadratic inequality, that is that the collection (10) is strictly complete with a quadratic function $S$.

(II) $\Longrightarrow(\mathrm{I})$. This implication directly follows from Lyapunov argument. Notice that the rank condition implies that $V$ is positive definite.

The main advantage of the Lyapunov function in the form (9) is that it allows us to give algebraic necessary and sufficient conditions for completeness of the collection (10) by the use of Farkas lemma instead of $\mathcal{S}$-procedure for quadratic forms which leads in general only to sufficient conditions. The main disadvantage of function (9) is that Theorem 2 says nothing about how large $M$ should be to satisfy condition (10). Moreover, even for linear systems the number $M$ can be significantly larger than its lower bound $n$ [10].

In order to give an algebraic criterion of stabilizability via controlled switching with an $\mathcal{H C}$-Lyapunov function we need the concept of strictly negative dominant diagonal matrices.

Definition 4 A square $M \times M$ real matrix $\Gamma$ is said to be a matrix with a strictly negative dominant diagonal if its entries satisfy the strict inequalities

$$
\gamma_{j j}+\sum_{s=1, s \neq j}^{M}\left|\gamma_{j s}\right|<0, \quad j=1, \ldots, M .
$$


Notice that if the matrix $\Gamma$ has a strictly negative dominant diagonal then it is Hurwitz. This fact immediately follows from Gerschgorin's theorem (see, e.g. [13]) about localization of eigenvalues of a square matrix.

In the sequel, for convenience we denote a convex combination of matrices $A_{r}, r \in \mathbb{N}$ by $\mathcal{A}$ :

$$
\mathcal{A}=\sum_{r=1}^{k} \tau_{r} A_{r}, \quad \tau_{r} \geq 0, \quad \sum_{r=1}^{k} \tau_{r}=1,
$$

where $A_{r}$ stands for $A+B K_{r}$, and $\mathcal{A}_{i}, \mathcal{A}_{j}$ stand for (perhaps equal) convex combinations of the matrices $A_{r}, r \in \mathbb{N}$.

Theorem 3 The following statements are equivalent:

I. There exists a switching function $\mathcal{I}: \mathbb{R}^{n} \rightarrow \mathbb{N}$ which exponentially stabilizes the system (6), (8), (7) with an $\mathcal{H C}$-Lyapunov function.

II. There exist a number $M \geq n$, a full rank $n \times M$ matrix $\mathcal{L}=\left(l_{1}, l_{2}, \ldots, l_{M}\right)$, an $M \times M$ matrix $\Gamma$ with a strictly negative dominant diagonal and $M$ convex combinations (11) $\mathcal{A}_{1}, \ldots, \mathcal{A}_{M}$ such that the following matrix equation is satisfied

$$
\left(\mathcal{A}_{1}^{\top} l_{1}, \ldots, \mathcal{A}_{M}^{\top} l_{M}\right)=\mathcal{L} \Gamma^{\top}
$$

Proof: The proof is based on the ideas of [10] and the following simple result which is a modification of the well known Farkas lemma [14].

Lemma 1 Consider the non-empty set $\Omega$ defined by the following system of linear inequalities

$$
a_{i}^{\top} x \leq 0, \quad i=1, \ldots, M, \quad x, a_{i} \in \mathbb{R}^{n} .
$$

and the collection of linear forms

$$
\left\{b_{1}^{\top} x, \ldots, b_{k}^{\top} x\right\}, \quad x, b_{j} \in \mathbb{R}^{n} .
$$

Then the following statements are equivalent:

i. The collection (13) is complete in $\Omega$.

ii. There exist nonnegative numbers $\alpha_{i} \geq 0, i=1, \ldots, M$ and $\beta_{j} \geq 0, j=$ $1, \ldots, k, \sum_{j=1}^{k} \beta_{j}=1$ such that

$$
\sum_{j=1}^{k} \beta_{j} b_{j}=\sum_{i=1}^{M} \alpha_{i} a_{i}
$$


Proof: $\quad(\mathrm{i}) \Longrightarrow$ (ii). Consider a mapping $\mathcal{N}: \Omega \rightarrow \mathbb{R}^{k}: x \mapsto\left(b_{1}^{\top} x, \ldots, b_{k}^{\top} x\right)^{\top}$. Obviously the set $\mathcal{N}(\Omega)$ is a convex cone. Since the collection $b_{j}^{\top} x$ is complete in $\Omega$ the cone $\mathcal{N}(\Omega)$ does not intersect the open positive orthant convex cone $\mathcal{P}$ in $\mathbb{R}^{k}$. Therefore by virtue of the Separation Theorem the cones $\mathcal{N}(\Omega)$ and $\mathcal{P}$ can be properly separated by the hyperplane passing through the origin (see [14, Theorem 11.7]). Thus there exists a nonzero vector $\beta \in \mathbb{R}^{k}$ such that

$$
\begin{array}{r}
\mathcal{N}(x)^{\top} \beta \leq 0 \quad \forall x \in \Omega \\
p^{\top} \beta \geq 0 \quad \forall p \in \mathcal{P} .
\end{array}
$$

It follows from (15) that all $k$ entries of $\beta=\left(\beta_{1}, \ldots, \beta_{k}\right)^{\top}$ are nonnegative and since $\beta \neq 0$ one can take $\beta_{j}, j=1, \ldots, k$ to satisfy $\beta_{1}+\ldots+\beta_{k}=1$. Therefore in the set $\Omega$ we have

$$
\left(\beta_{1} b_{1}+\ldots+\beta_{k} b_{k}\right)^{\top} x \leq 0
$$

The result now follows immediately from Farkas' lemma (see [14, Corollary 22.3.1])

(i) (ii) It is a consequence of Farkas' lemma.

$(\mathrm{I}) \Longrightarrow(\mathrm{II})$. From the previous theorem we know that (I) implies the existence of a function $V: \mathbb{R}^{n} \rightarrow \mathbb{R}_{+}$of the form (9) such that for the closed loop system it satisfies

$$
\lambda_{1}|x|^{2} \leq V(x) \leq \lambda_{2}|x|^{2}, \quad \lambda_{1}, \lambda_{2}>0
$$

and

$$
\dot{V}(x) \leq-2 \varepsilon V(x), \quad \varepsilon>0 .
$$

Denote $A_{r}(\varepsilon)=A_{r}+\varepsilon I_{n}, r \in \mathbb{N}$, where $\varepsilon$ comes from the estimate (16). Consider the following sets

$$
\Omega_{\nu}=\left\{x \in \mathbb{R}^{n}:\left(l_{\nu}^{\top} x\right)^{2} \geq\left(l_{j}^{\top} x\right)^{2}, \quad j=1, \ldots, M\right\},
$$

$\nu=1, \ldots, M$. For any $\nu \in\{1, \ldots, M\}$ the set $\Omega_{\nu}$ can be split into the two subsets $\Omega_{\nu}^{+}$and $\Omega_{\nu}^{-}$, where

$$
\begin{gathered}
\Omega_{\nu}^{+}=\left\{x \in \mathbb{R}^{n}:\left(l_{\nu}^{\top} x\right) \geq\left|l_{j}^{\top} x\right|, \quad j=1, \ldots, M\right\}, \\
\Omega_{\nu}^{-}=\left\{x \in \mathbb{R}^{n}:\left(l_{\nu}^{\top} x\right) \leq-\left|l_{j}^{\top} x\right|, \quad j=1, \ldots, M\right\} .
\end{gathered}
$$

It is clear also that

$$
\bigcup_{\nu}\left\{\Omega_{\nu}^{+} \cup \Omega_{\nu}^{-}\right\}=\mathbb{R}^{n}
$$


Therefore (16) is satisfied as long as for all $\nu=1, \ldots, M$ the collection

$$
\left\{\left(l_{\nu}^{\top} x\right)\left(l_{\nu}^{\top} A_{1}(\varepsilon) x\right), \ldots,\left(l_{\nu}^{\top} x\right)\left(l_{\nu}^{\top} A_{k}(\varepsilon) x\right)\right\}
$$

is complete in $\Omega_{\nu}$, or, equivalently, the collection

$$
\left\{l_{\nu}^{\top} A_{1}(\varepsilon) x, \ldots, l_{\nu}^{\top} A_{k}(\varepsilon) x\right\}
$$

is complete in $\Omega_{\nu}^{+}$. Notice that each inequality $l_{\nu}^{\top} x \geq\left|l_{j}^{\top} x\right|$ is equivalent to two linear inequalities

$$
\left(-l_{\nu}+l_{j}\right)^{\top} x \leq 0 \quad \text { and } \quad\left(-l_{\nu}-l_{j}\right)^{\top} x \leq 0
$$

Therefore applying lemma 1 one can conclude that there exist $M$ convex combinations $\mathcal{A}_{1}, \ldots, \mathcal{A}_{M}$ such that

$$
\mathcal{A}_{\nu}^{\top} l_{\nu}=\sum_{j=1}^{M} d_{\nu j} l_{j}-\varepsilon l_{\nu}, \quad \nu=1, \ldots, M
$$

where the numbers $d_{\nu j}$ satisfy

$$
d_{\nu \nu}+\sum_{j=1, j \neq \nu}^{M}\left|d_{\nu j}\right| \leq 0 .
$$

The relation (18) can be rewritten in the form

$$
\mathcal{A}_{\nu}^{\top} l_{\nu}=\sum_{j=1}^{M} \gamma_{\nu j} l_{j}, \quad \nu=1, \ldots, M,
$$

where

$$
\gamma_{\nu \nu}+\sum_{j=1, j \neq \nu}^{M}\left|\gamma_{\nu j}\right|<0
$$

In matrix notations (19) can be rewritten in the form (12), where the matrix $\Gamma$ has strictly negative dominant diagonal.

$(\mathrm{II}) \Longrightarrow(\mathrm{I})$. In the proof of the implication (I) $\Longrightarrow($ II) we have established that (II) is equivalent to the completeness of the collection (17) in $\Omega_{\nu}$. Let $i(x)$ be an index such that for all $\nu \in\{1, \ldots, M\}$

$$
\left(l_{\nu}^{\top} x\right)\left(l_{\nu}^{\top} A_{i} x\right) \leq-\varepsilon\left(l_{\nu}^{\top} x\right)^{2}=-\varepsilon V(x)
$$

then the switching function $\mathcal{I}(x)=i(x)$ stabilizes the system at the origin. 
In $[10$, Theorem 2] it was shown that the equation

$$
A^{\top} \mathcal{L}=\mathcal{L} \Gamma^{\top}
$$

with above mentioned assumptions on $\mathcal{L}$ and $\Gamma$ is solvable if and only if the matrix $A$ is Hurwitz. This fact allows one to formulate the following consequence of Theorem 3 which gives a computational criterion of the stabilizability via controlled switching.

Corollary 1 If there exists a fixed controller consisting of a convex combination of the basic controllers (7) which stabilizes the system (6) then the system (6) is stabilizable via controlled switching with the basic controllers (7).

Proof: $\quad$ Take $\mathcal{A}=\mathcal{A}_{1}=\mathcal{A}_{2}=\ldots=\mathcal{A}_{M}$. Then (12) becomes $\mathcal{A}^{\top} \mathcal{L}=\mathcal{L} \Gamma^{\top}$ which is solvable iff $\mathcal{A}$ is Hurwitz.

This statement was established in [3] as a sufficient condition of quadratic stabilizability of linear system via controlled switching. Therefore, if the hypothesis of Corollary 1 holds then there exists a quadratic form which can be taken as a Lyapunov function for the closed loop system.

The class of $\mathcal{H C}$-Lyapunov functions is convenient for analysis/design of hybrid systems when we a priori know that the switching function satisfies the condition $\mathcal{I}(\tau x)=\mathcal{I}(x), \forall \tau \neq 0$, and when it is desired that the sets of the form $\left\{x \in \mathbb{R}^{n}: V(x) \leq C, C>0\right\}$ are convex. The latter requirement is convenient when one solves the problem of stabilization via controlled switching under constraints on state variables given in a form of a convex set.

Now let us briefly discuss a more general class of Lyapunov functions which can be nonconvex, however we still assume that they are homogeneous.

\subsection{Homogeneous Lyapunov functions}

In some examples of analysis/design of hybrid systems [3, 4] it is a priori known that the switching function is constant in some cones of the phase space and invariant under central symmetric transform. In this case among the set of all Lyapunov functions which prove exponential stabilizability of such hybrid systems it is sufficient to take into account only homogeneous with degree two Lyapunov functions. Let us consider piecewise quadratic homogeneous functions of the form

$$
V(x)=\max _{1 \leq j \leq M}\left\{x^{\top} L_{1} x, \ldots, x^{\top} L_{M} x\right\}
$$


where $L_{j}=L_{j}^{\top}, j=1, \ldots, M$ are some symmetric matrices such that the collection

$$
\left\{-x^{\top} L_{1} x, \ldots,-x^{\top} L_{M} x\right\}
$$

is strictly complete. Notice that the strict completeness of the collection (23) implies positive definiteness of the function $V$ and therefore it can be considered as a Lyapunov function candidate. Along with the function (22) one can consider the following Lyapunov function candidate

$$
V(x)=\min _{1 \leq j \leq M}\left\{x^{\top} L_{1} x, \ldots, x^{\top} L_{M} x\right\}
$$

where the collection (23) is again assumed to be strictly complete. It is not difficult to notice that if for all $i=1, \ldots, M$ the collections

$$
\left\{x^{\top}\left(A_{1}^{\top} L_{i}+L_{i} A_{1}\right) x, \ldots, x^{\top}\left(A_{k}^{\top} L_{i}+L_{i} A_{k}\right) x\right\}
$$

are strictly complete in the sets

$$
\Omega_{i}=\left\{x \in \mathbb{R}^{n}: x^{\top} L_{i} x \geq x^{\top} L_{j} x, \quad j=1, \ldots, M, j \neq i\right\}
$$

then the system is stabilizable via controlled switching with Lyapunov function (22). By analogy, the function (24) proves stabilizability via controlled switching if and only if the collections (25) are strictly complete in

$$
\Omega_{i}=\left\{x \in \mathbb{R}^{n}: x^{\top} L_{i} x \leq x^{\top} L_{j} x, \quad j=1, \ldots, M, j \neq i\right\} .
$$

\section{On Passification via Controlled Switching}

In this section we will discuss passifiability issues via controlled switching and by using results of the previous section on existence of a Lyapunov function

we will present an algebraic criterion of passifiability via controlled switching for linear systems with linear basic controllers.

Definition 5 A system (2) has the KYP (Kalman-Yakubovich-Popov) (resp. state strict $K Y P$, output strict $K Y P$ ) property if there exists a nonnegative function $V: \mathbb{R}^{n} \rightarrow \mathbb{R}_{+}, V(0)=0$, such that the collection

$$
\left\{L_{f+g \phi_{1}} V(x), L_{f+g \phi_{2}} V(x), \ldots, L_{f+g \phi_{k}} V(x)\right\}
$$

is complete (resp. strictly complete, strictly complete with respect to the mapping $h$ ) and the following relation holds for all $x \in \mathbb{R}^{n}$

$$
L_{g_{1}} V(x)=h(x)^{\top} .
$$


The Definition 5 can be considered as an alternative definition of passifiable systems. The following result can be proved similarly to [15].

Proposition 1 The following statements are equivalent:

I. The system (2) has the KYP property (resp. state strict KYP property, output strict KYP property).

II. The system (2) is passifiable (resp. state strictly passifiable, output strictly passifiable) via controlled switching with the basic controllers (3) with a storage function which has derivatives in all directions.

Furthermore, suppose that condition (I) holds and let $i(x)$ be an index such that $x \in \Omega_{i}$. Then the switching function $\mathcal{I}(x)=i(x)$ passifies (resp. state strictly passifies, output strictly passifies) the system (2).

Additionally, if the system (2) is strictly passifiable with a positive definite radially unbounded storage function then the switching function $\mathcal{I}(x)=i(x)$ globally stabilizes the system at the origin for $v \equiv 0$.

The introduced concept of passifiability allows one to employ well-known results such as passivity theorem, small-gain theorem, $\mathcal{L}_{2}$-gain disturbance attenuation (see, e.g. [11]) for a wide class of hybrid systems.

Notice that in practical problems it might be quite difficult to establish that the collection (26) is complete. This condition can be replaced by a stronger condition which is sometimes easier to check. Assume that there exists a set of nonnegative numbers $\tau_{r} \geq 0, r \in \mathbb{N}, \sum_{r=1}^{k} \tau_{r}>0$ such that

$$
\sum_{r=1}^{k} \tau_{r} L_{f+g \phi_{r}} V(x) \leq-S(x),
$$

where $S$ is nonnegative (resp. positive definite, $S=S_{1} \circ h$ with positive definite $S_{1}$ ). It is obvious that if $(28)$ is valid then the collection (26) is complete (resp. strictly complete, strictly complete with respect to mapping $h$ ). This trick is known as the $\mathcal{S}$-procedure. The converse statement is (generally speaking) not true. However, if the mapping $\mathcal{N}: \mathbb{R}^{n} \rightarrow$ $\mathbb{R}^{k+1}: x \mapsto\left(L_{f+g \phi_{1}} V(x), \ldots, L_{f+g \phi_{k}} V(x), S(x)\right)^{\top}$ has an image of $\mathbb{R}^{n}$ that is convex in $\mathbb{R}^{k+1}$ then the statement $(28)$ is equivalent to strict completeness of the collection (26). This can be proved by a standard technique based on the Separation Theorem. This technique was used by Yakubovich in the proof of losslessness of the $\mathcal{S}$-procedure for two quadratic functions over the real linear space [16]. The case of two quadratic forms is known as Finsler's theorem (see survey [17]). 
It is interesting to notice that the collection (26) can be complete and the expression (28) can be satisfied even when among the set of basic controllers there are no single passifiable controller. In this case the system (2) can be passified only by an appropriate switching rule.

From the theory of dissipative systems it is known that an alternative definition of passive systems can be done by using the concept of positive realness. With this in mind we give a definition of a positive real system via controlled switching.

Definition 6 The system (2), (4), (3) is said to be positive real (resp. strictly positive real) via controlled switching if there exist a switching function $\mathcal{I}: \mathbb{R}^{n} \rightarrow \mathbb{N}$ and a nonnegative (resp. positive definite) function $S: \mathbb{R}^{n} \rightarrow \mathbb{R}_{+}$such that for the closed loop system for all $v \in \mathcal{V}$ and $0 \leq t<T_{x_{0}, v, \mathcal{I}}$ we have

$$
\int_{0}^{t} h(x(s))^{\top} v(s) d s \geq \int_{0}^{t} S(x(s)) d s \geq 0
$$

whenever $x(0)=0$.

It is clear that if the system is (state strictly) passifiable via controlled switching then it is (strictly) positive real via controlled switching. Indeed, from the dissipation inequality (5) by using nonnegativity of $V(x)$ for all $x \in \mathbb{R}^{n}$ and $V(0)=0$ we immediately obtain (29). The converse statement, in general, is not true. However, it is true if the available storage function

$$
V_{a}(x)=\min _{i} \sup _{v \in \mathcal{V}, t \geq 0}\left\{\int_{0}^{t}\left(S(x(s))-h(x(s))^{\top} v\right) d s\right\},
$$

where $x(0)=x$, is finite for all $x \in \mathbb{R}^{n}$ (see, e.g. $[15,19,11]$ ). Here minimum is taken over all switching functions which make the closed loop system (strictly) positive real. For systems without switchings the available storage is finite for all $x$ as long the state $x$ is reachable from the origin $[15,11]$ ). For hybrid systems this statement cannot be true in general, since it is possible that the switching function which makes all states reachable from the origin does not make the system positive real. This simple observation shows that the concepts of reachability/controllability are of utmost importance for the problems of analyzing passivity properties of hybrid systems.

Now as an example consider the problem of passification of the following linear system

$$
\left\{\begin{array}{l}
\dot{x}=A x+B u+D v \\
y=C x
\end{array}\right.
$$


via controlled switching with the basic controllers

$$
u_{1}=K_{1} x, u_{2}=K_{2} x, \ldots, u_{k}=K_{k} x .
$$

Note that since the closed loop system satisfies the global Lipschitz condition for $v=0$ and $D$ is a constant matrix we have $T_{x_{0}, v, \mathcal{I}}=\infty$.

Taking $V(x)=x^{\top} P x$ from Proposition 1 one can conclude that the system (30) is passifiable with a quadratic storage function via controlled switching as long as the collection of quadratic forms

$$
\begin{array}{r}
\left\{x^{\top}\left(\left(A+B K_{1}\right)^{\top} P+P\left(A+B K_{1}\right)\right) x, \ldots,\right. \\
\left.x^{\top}\left(\left(A+B K_{k}\right)^{\top} P+P\left(A+B K_{k}\right)\right) x\right\}
\end{array}
$$

is complete and

$$
P D=C^{\top}
$$

In order to find sufficient conditions of passifiability we replace condition (32) with a stronger one

$$
\sum_{r=1}^{k} \tau_{r}\left(A_{r}^{\top} P+P A_{r}\right) \leq 0
$$

for some $\tau_{r} \geq 0, \sum_{r=1}^{k} \tau_{r}>0$, where $A_{r}=A+B K_{r}, r \in \mathbb{N}$.

Theorem 4 Assume that there exist numbers $\tau_{r} \geq 0, \sum_{r=1}^{k} \tau_{r}>0$ so that the matrix $\mathcal{A}=\sum_{r=1}^{k} \tau_{r} A_{r}$ is Hurwitz and the pairs $(\mathcal{A}, D),(\mathcal{A}, C)$ are controllable and observable respectively. Furthermore, suppose that the following frequency domain inequality

$$
\operatorname{Re} C\left(i \omega I_{n}-\mathcal{A}\right)^{-1} D \geq 0, \quad i^{2}=-1
$$

is satisfied for all $\omega \in \mathbb{R}$ with

$$
\operatorname{det}\left(i \omega I_{n}-\mathcal{A}\right) \neq 0 \text {. }
$$

Then the system (30) is passifiable via controlled switching with basic controllers (31).

The proof of this theorem immediately follows from frequency domain conditions of solvability of nonstrict Lyapunov matrix inequalities. See e.g. [18]. Notice that the frequency domain inequality means positive realness of the transfer function.

In state space representations this condition means that for some $\tau_{r} \geq$ $0, \sum_{r=1}^{k} \tau_{r}>0$ the system

$$
\left\{\begin{array}{l}
\dot{x}=\sum_{r=1}^{k} \tau_{r}\left(A+B K_{r}\right)+D v \\
y=C x
\end{array}\right.
$$


is weakly minimum-phase and $\operatorname{det} C D \neq 0$.

It is worth mentioning that although the frequency domain inequality gives necessary and sufficient conditions of solvability of Lyapunov inequalities, the result of Theorem 4 can be conservative. The reason is that we replaced the condition on the completeness of a collection of quadratic forms by positive semidefiniteness of their convex combination ( $\mathcal{S}$-procedure). The second reason is that the storage function which proves passivity in this case is a quadratic form which may be too restrictive. However, this drawback can be overcome if we use the result derived in the previous section on the existence of piecewise quadratic Lyapunov functions and the nonlinear version of Kalman-Yakubovich-Popov lemma (Proposition 1). In this case solvability of equations (12) and

$$
l_{1} B^{\top} l_{1}=\ldots=l_{M} B^{\top} l_{M}=C
$$

implies state strict passifiability via controlled switching with the basic controllers (3).

Now let us briefly discuss the problem of feedback equivalence to a passive system via controlled switching.

Definition 7 The system (2) is said to be globally equivalent to a passive system via state feedback and controlled switching with the basic controllers (3) if there exist a switching function $\mathcal{I}: \mathbb{R}^{n} \rightarrow \mathbb{N}$ and a feedback

$$
v=\alpha(x)+\beta(x) w,
$$

where $\alpha: \mathbb{R}^{n} \rightarrow \mathbb{R}^{l}, \alpha(0)=0$ and $\beta: \mathbb{R}^{n} \rightarrow \mathbb{R}^{l \times l}, w(t) \in \mathbb{R}^{l}$ is a new input, such that the closed loop system is passive from $w$ to $y$.

Similar definitions can be given for feedback equivalence to a state or output strictly passive system. Notice that feedback equivalence can be local, global or, say, semiglobal, depending on the region in $\mathbb{R}^{n}$ where the dissipation inequality holds.

It is also interesting to study the case when the feedback in (36) depends only on output variable: $\alpha=\alpha_{1} \circ h, \beta=\beta_{1} \circ h$ and when the switching function also allows the following factorization $\mathcal{I}=\mathcal{I}_{1} \circ h$. In this case the system (2) will be referred to as equivalent to a passive system via output feedback and controlled switching.

By analogy, if there exists a switching function such that the closed loop system is minimum phase the system will be referred to as minimumphasable via controlled switching.

From [19] one can conclude that the system is locally state feedback equivalent to a passive system via controlled switching if it is weakly minimumphasable and has relative degree one at the origin. 
It is known that controllable linear system $(A, B, C)$ with a full rank matrix $B$ is state feedback equivalent to a state strictly passive system if it is minimum phase and $C B$ is nonsingular [20]. Under the same conditions the system is output feedback equivalent to a state strictly passive system [21]. That is, that for linear systems properties of state feedback and output feedback strict state passivity are equivalent (similar semiglobal statement holds also for nonlinear systems in case of exponential minimumphaseness [21]). So one may conjecture that similar equivalence is retained for piecewise linear systems with controlled switching. However this is not the case. Indeed, in case of state feedback it is possible that among the set of basic controllers there is no controller which makes the system minimum phase. However, in case of output feedback there must exist at least one basic controller which makes the system minimum phase (it directly follows from the required factorization $\mathcal{I}=\mathcal{I}_{1} \circ h$ ). Thus for linear systems with linear basic controllers if the system is output feedback passive via controlled switching then it is output feedback passive without switchings.

In case of nonlinear systems, however, the switchings may be useful even for problems of output feedback passification. For example, the system

$$
\left\{\begin{array}{l}
\dot{x}=-x \\
\dot{y}=y+u+v
\end{array}\right.
$$

with basic controllers

$$
u_{1}=-y+y^{2}, \quad u_{2}=-y-y^{2}
$$

is not globally output feedback equivalent to a passive system with a linear feedback

$$
v=-K y+w
$$

for one particular basic controller. However via controlled switching with switching function which depends only on $y$ it can be made passive for arbitrarily nonnegative $K$.

\section{Conclusion}

In this paper we have given criteria of stabilizability of systems via controlled switching in terms of piecewise quadratic Lyapunov functions. Numerical complexity of the search for Lyapunov functions, particularly in the problems of designing the switching rules, can be extremely high. With this in mind we presented sufficient conditions of stabilizability via controlled switching for different classes of Lyapunov functions. This can reduce computational costs 
when it is desired to find an appropriate Lyapunov function from one of the given classes. Note that the presented conditions in Theorem 3, Corollary 1, and Theorem 4 are bilinear matrix inequalities (BMI:s).

Based on the results on stabilizability some criteria of passifiability via controlled switching have also been presented. This concept is of utmost importance for the problem of designing hybrid systems with $\mathcal{L}_{2}$ gain. It allows to reduce the computational costs in the design of hybrid systems with some structured (cascade and/or feedback interconnections) properties since the corresponding BMI:s will be of smaller dimension. In this case the search for Lyapunov functions can be performed independently for each subsystem.

The concepts of controllability and reachability via controlled switching are important for establishing connections between passifiability and positive realness via controlled switching. This will be a topic of further work.

The results on passifiability of a system through switching might be used in a recursive manner to establish passivity of successively larger parts of a hybrid system with a hierarchical structure. This would be an interesting problem for future investigations.

\section{References}

[1] M.S. Branicky, "Stability of switched hybrid systems", In Proceedings of the 33rd Conference on Decision and Control, 1994, Lake Buena Vista, FL, USA, pp. 3498-3503.

[2] A.V. Savkin, I.R. Petersen, E. Skafidas And R.J. Evans, "Hybrid dynamical systems: robust control synthesis problems," Systems \& Control Letters, vol. 29, 1996, pp. 81-90.

[3] E. Skafidas, I.R. Petersen, R.J. Evans and A.V. Savkin, "Quadratic stabilizability of state feedback hybrid control systems", in Proc. of The Fourth International Conference on Control, Automation, Robotics and Vision (ICARCV'96), Singapore, 1996, pp. 1073-1077.

[4] M. Johanson and A. Rantzer, "Computation of piecewise quadratic Lyapunov functions for hybrid systems", Technical report ISSN 0280-5316 ISRN LUTFD2/TFRT-7549-SE, Lund Institute of Technology, 1996, to appear in IEEE Transaction on Automatic Control, special issue on hybrid systems, April, 1998.

[5] S.V. Emelyanov, Automatic Control Systems with Variable Structure, Moskow-Nauka, 1967, in Russian.

[6] M.S. Branicky, V.S. Borkar and S.K. Mitter, "A unified framework for hybrid control", In Proceedings of the 33rd Conference on Decision and Control, 1994, Lake Buena Vista, FL, USA, pp. 4228-4234. 
[7] S. Boyd, L.E. Ghaoui, E. Feron, and V. Balakrishnan, Linear Matrix Inequalities in System and Control Theory, Studies in Applied Mathematics, vol. 15, SIAM, Philadelphia, 1994.

[8] Y. Nesterov and A. Nemirovski, Interior point polynomial methods in convex programming, Studies in Applied Mathematics, vol. 13, SIAM, Philadelphia, 1993.

[9] A.P. Molchanov and E.S. Pyatnitskii, "Lyapunov functions that specify necessary and sufficient conditions of absolute stability of nonlinear nonstationary control systems, Part I", Automation and Remote Control, vol. 47, 1986, pp. 344354.

[10] A.P. Molchanov and E.S. Pyatnitskii, "Lyapunov functions that specify necessary and sufficient conditions of absolute stability of nonlinear nonstationary control systems, Part I", Automation and Remote Control, vol. 47, 1986, pp. 620630.

[11] A.J. van der Schaft, $\mathcal{L}_{2}$-gain and passivity techniques in nonlinear control, Lecture Notes on Control and Information Sciences, Springer, London, 1996

[12] N.N. Krasovskii Stability of motion. (Stanford Univ.Press), 1963; (in Russian: 1959).

[13] G.W. Stewart, Ji-Guang Sun, Matrix Perturbation Theory, Academic Press, 1990.

[14] R.T. Rockafellar, Convex Analysis, Princeton, Princeton University Press, Second Printing, 1972.

[15] D.J. Hill, and P.J. Moylan, "The stability of nonlinear dissipative systems", IEEE Trans. Aut. Contr., vol. 21, 1976, pp. 708-711.

[16] V.A. Yakubovich, "S-procedure in nonlinear control theory," Vestnik Leningradskogo Universiteta, Seria Matematika, 1971, pp. 62-71, in Russian.

[17] F. Uhlig, "A recurring theorem about pairs of quadratic forms and extensions: a survey", Linear algebra and its applications, vol. 25, 1979, pp. 219-237.

[18] A. Kh. Gelig, G.A. Leonov and V.A. Yakubovich, The stability of nonlinear systems with a nonunique equilibrium state, Moskow-Nauka, 1978, in Russian.

[19] C.I. Byrnes, A. Isidory and J.C. Willems, "Passivity, feedback equivalence and the global stabilization of minimum phase nonlinear systems", IEEE Trans. Aut. Contr., vol. 10, 1991, pp. 1122-1137.

[20] A. Saberi, P.V. Kokotovic and H.J. Sussman, "Global stabilization of partially linear composite systems," SIAM Journal Control Optimization, vol. 28, 1990, pp. 1491-1503.

[21] A.L. Fradkov, and D.J. Hill, "Exponential feedback passivity and stabilizability of nonlinear systems", Technical report EE9357, Sydney University, Australia, 1993. 